\title{
Partial Dynamical Symmetry in Nuclear Systems
}

\author{
J. Escher
}

This article was submitted to: Computational and Group Theoretical Methods in Nuclear Physics, Playa del Carmen, Mexico 2/18/2003 - 2/21/2003

\section{June 2, 2003}

U.S. Department of Energy

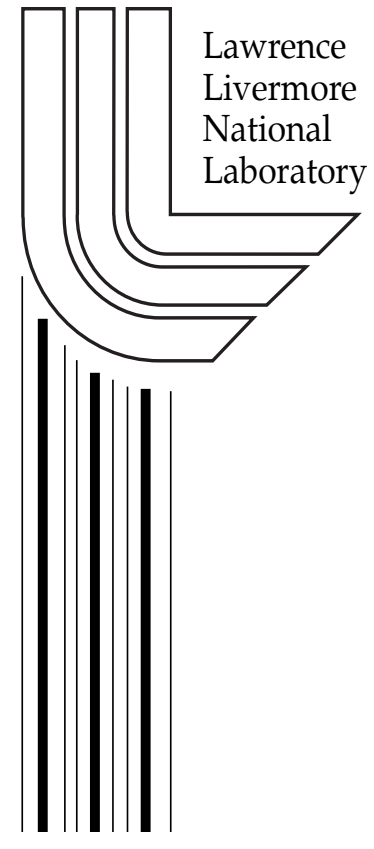




\section{DISCLAIMER}

This document was prepared as an account of work sponsored by an agency of the United States Government. Neither the United States Government nor the University of California nor any of their employees, makes any warranty, express or implied, or assumes any legal liability or responsibility for the accuracy, completeness, or usefulness of any information, apparatus, product, or process disclosed, or represents that its use would not infringe privately owned rights. Reference herein to any specific commercial product, process, or service by trade name, trademark, manufacturer, or otherwise, does not necessarily constitute or imply its endorsement, recommendation, or favoring by the United States Government or the University of California. The views and opinions of authors expressed herein do not necessarily state or reflect those of the United States Government or the University of California, and shall not be used for advertising or product endorsement purposes.

This is a preprint of a paper intended for publication in a journal or proceedings. Since changes may be made before publication, this preprint is made available with the understanding that it will not be cited or reproduced without the permission of the author.

This work was performed under the auspices of the United States Department of Energy by the University of California, Lawrence Livermore National Laboratory under contract No. W-7405-Eng-48.

This report has been reproduced directly from the best available copy.

Available electronically at http://www.doc.gov/bridge

Available for a processing fee to U.S. Department of Energy

And its contractors in paper from

U.S. Department of Energy

Office of Scientific and Technical Information

P.O. Box 62

Oak Ridge, TN 37831-0062

Telephone: (865) 576-8401

Facsimile: (865) 576-5728

E-mail: reports@adonis.osti.gov

Available for the sale to the public from

U.S. Department of Commerce

National Technical Information Service

5285 Port Royal Road

Springfield, VA 22161

Telephone: (800) 553-6847

Facsimile: (703) 605-6900

E-mail: orders@ntis.fedworld.gov

Online ordering: http://www.ntis.gov/ordering.htm

OR

Lawrence Livermore National Laboratory

Technical Information Department's Digital Library

http://www.llnl.gov/tid/Library.html 


\title{
PARTIAL DYNAMICAL SYMMETRY IN NUCLEAR SYSTEMS
}

\author{
JUTTA ESCHER \\ Nuclear Theory and Modeling Group, $N$ Division \\ Lawrence Livermore National Laboratory, \\ P.O. Box 808, L-414, Livermore, CA 94551, USA \\ E-mail: escher1@llnl.gov
}

\begin{abstract}
Partial dynamical symmetry (PDS) extends and complements the concepts of exact and dynamical symmetry. It allows one to remove undesired constraints from an algebraic theory, while preserving some of the useful aspects of a dynamical symmetry, and to study the effects of symmetry breaking in a controlled manner. An example of a PDS in an interacting fermion system is presented. The associated PDS Hamiltonians are closely related with a realistic quadrupole-quadrupole interaction and provide new insights into this important interaction.
\end{abstract}

\section{Introduction}

Algebraic, symmetry-based theories provide an elegant and practical approach for describing and understanding a variety of physical systems. These theories offer the greatest simplifications when the interaction under consideration is symmetry preserving in the selected state labeling scheme, that is, when the Hamiltonian either commutes with all the generators of a particular group ('exact symmetry') or when it is written in terms of and commutes with the Casimir operators of a chain of nested groups ('dynamical symmetry'). An exact or dynamical symmetry not only facilitates the numerical treatment of the Hamiltonian, but also its interpretation and thus provides considerable insight into the physics of a given system.

Algebraic models can also be of value in situations where it is necessary to introduce symmetry-breaking terms in the Hamiltonian in order to achieve agreement with experimentally observed features. Pragmatically, it is often possible to decompose the offending terms into basic parts ("irreducible tensor operators") which exhibit specific transformation properties. Provided the appropriate group coupling coefficients and the matrix elements of some elementary tensor operators are available, matrix elements 
of operators that connect inequivalent irreducible representations can be determined and the exact eigenvalues and eigenstates can then be obtained (at least in principle). Furthermore, by studying the effects of symmetrybreaking terms, one gains new insights into the dynamics of the system, the relevance and robustness of the symmetries under consideration, and their limitations. In some cases new symmetries emerge from a broken-symmetry regime. Their identification and interpretation is often simplified in the framework of an algebraic model. Examples of such emerging symmetries include quasi-dynamical symmetry ${ }^{1}$ and pseudo-spin symmetry ${ }^{2}$.

One can also consider introducing some intermediate structure that allows for symmetry breaking but retains some aspects of the dynamical symmetry. Partial dynamical symmetry (PDS) provides such a structure ${ }^{3}$. Two types of partial dynamical symmetry have been studied in recent years. Partial dynamical symmetry of the first kind preserves the advantages of a dynamical symmetry for a part of the system. It corresponds to a particular symmetry breaking for which the Hamiltonian is not invariant under the symmetry group and hence various irreducible representions (irreps) are mixed in its eigenstates, yet it possesses a subset of 'special' solvable states which respect the symmetry. PDS of the second kind corresponds to a symmetry breaking for which all eigenstates of the Hamiltonian preserve part of the dynamical symmetry ${ }^{4}$. In this scenario, the dynamical symmetry associated with an intermediate group $G_{2}$ in a subchain $G_{1} \supset G_{2} \supset G_{3}$ is broken for all states of the system, while the remaining (dynamical) symmetries are preserved. The resulting Hamiltonian is in general not analytically solvable, but its eigenstates can still be (partly) classified by quantum labels associated with the groups $G_{1}$ and $G_{3}$. Further generalizations of the partial symmetry concept have been considered as well ${ }^{5}$.

This contribution will discuss an example of a partial dynamical symmetry in an interacting fermion system. More specifically, in the framework of the symplectic shell model (SSM), there exists a family of PDS Hamiltonians which are closely related to the nuclear quadrupole-quadrupole interaction. The Hamiltonians and their eigenstates are discussed and applications to the deformed light nuclei ${ }^{20} \mathrm{Ne}$ and ${ }^{24} \mathrm{Mg}$ are presented.

\section{Quadrupole-Quadrupole Interaction in the SSM}

The quadrupole-quadrupole interaction is an important ingredient in models that aim at reproducing quadrupole collective properties of nuclei. A model which is able to fully accommodate the action of the collec- 
tive quadrupole operator, $Q_{2 m}=\sqrt{\frac{16 \pi}{5}} \sum_{s} r_{s}^{2} Y_{2 m}\left(\hat{r}_{s}\right)$, is the symplectic shell model (SSM), an algebraic scheme which respects the Pauli exclusion principle ${ }^{6}$. In the SSM, this operator takes the form $Q_{2 m}=$ $\sqrt{3}\left(\hat{C}_{2 m}^{(11)}+\hat{A}_{2 m}^{(20)}+\hat{B}_{2 m}^{(02)}\right)$, where $\hat{A}_{l m}^{(20)}, \hat{B}_{l m}^{(02)}$, and $\hat{C}_{l m}^{(11)}$ are symplectic generators with good $\mathrm{SU}(3)$ [superscript $(\lambda, \mu)$ ] and $\mathrm{SO}(3)$ [subscript $l, m$ ] tensorial properties. The $\hat{A}_{l m}^{(20)}\left(\hat{B}_{l m}^{(02)}\right), l=0$ or 2 , create (annihilate) $2 \hbar \omega$ excitations in the system. The $\hat{C}_{l m}^{(11)}, l=1$ or 2 , generate a $\mathrm{SU}(3)$ subgroup and act only within one harmonic oscillator (h.o.) shell $\left(\sqrt{3} \hat{C}_{2 m}^{(11)}=\right.$ $Q_{2 m}^{E}$, the symmetrized quadrupole operator of Elliott, which does not couple different h.o. shells ${ }^{7}$, and $\hat{C}_{1 m}^{(11)}=\hat{L}_{m}$, the orbital angular momentum operator). A fermion realization of these generators has been given ${ }^{8}$.

A basis for the symplectic model is generated by applying symmetrically coupled products of the $2 \hbar \omega$ raising operator $\hat{A}^{(20)}$ with itself to the usual $0 \hbar \omega$ many-particle shell-model states. Each $0 \hbar \omega$ starting configuration is characterized by the distribution of oscillator quanta into the three cartesian directions, or, equivalently, by its $\mathrm{U}(1) \times \mathrm{SU}(3)$ quantum numbers $N_{\sigma}\left(\lambda_{\sigma}, \mu_{\sigma}\right)$. Here $\left(\lambda_{\sigma}, \mu_{\sigma}\right)$ are the Elliott $\mathrm{SU}(3)$ labels, and $N_{\sigma}=\sigma_{1}+\sigma_{2}+\sigma_{3}$ is related to the eigenvalue of the oscillator number operator. ${ }^{20} \mathrm{Ne}$, for instance, has $N_{\sigma}=48.5$ (after removal of the centerof-mass contribution) and $\left(\lambda_{\sigma}, \mu_{\sigma}\right)=(8,0)$. For ${ }^{24} \mathrm{Mg}$, one finds $N_{\sigma}=62.5$ and $\left(\lambda_{\sigma}, \mu_{\sigma}\right)=(8,4)$. The product of $N / 2$ raising operators $\hat{A}^{(20)}$ generates $N \hbar \omega$ excitations for each starting irrep $N_{\sigma}\left(\lambda_{\sigma}, \mu_{\sigma}\right)$. Each such product operator $P^{N\left(\lambda_{n}, \mu_{n}\right)}$, labeled according to its $\mathrm{SU}(3)$ content, $\left(\lambda_{n}, \mu_{n}\right)$, is coupled with $\left|N_{\sigma}\left(\lambda_{\sigma}, \mu_{\sigma}\right)\right\rangle$ to good $\mathrm{SU}(3)$ symmetry $\rho(\lambda, \mu)$, with $\rho$ denoting the multiplicity of the coupling $\left(\lambda_{n}, \mu_{n}\right) \otimes\left(\lambda_{\sigma}, \mu_{\sigma}\right)$. To complete the basis state labeling, additional quantum numbers $\alpha=\kappa L M$ are required, where $L$ denotes the angular momentum with projection $M$, and $\kappa$ is a multiplicity index, which enumerates multiple occurrences of a particular $L$ value in the $\mathrm{SU}(3)$ irrep $(\lambda, \mu)$. The group chain corresponding to this labeling scheme is $\mathrm{Sp}(6, \mathrm{R}) \supset \mathrm{SU}(3) \supset \mathrm{SO}(3)$ which defines a dynamical symmetry basis.

The quadrupole-quadrupole interaction connects h.o. states differing in energy by $0 \hbar \omega, \pm 2 \hbar \omega$, and $\pm 4 \hbar \omega$, and may be written as

$$
\begin{aligned}
Q_{2} \cdot Q_{2}= & 9 \hat{C}_{S U 3}-3 \hat{C}_{S p 6}+\hat{H}_{0}^{2}-2 \hat{H}_{0}-3 \hat{L}^{2}-6 \hat{A}_{0} \hat{B}_{0} \\
& +\{\text { terms coupling different h.o. shells }\}
\end{aligned}
$$

where $\hat{C}_{S U 3}$ and $\hat{C}_{S p 6}$ are the quadratic Casimir invariants of $\mathrm{SU}(3)$ and $\operatorname{Sp}(6, \mathrm{R})$ with eigenvalues $2\left(\lambda^{2}+\mu^{2}+\lambda \mu+3 \lambda+3 \mu\right) / 3$ and $2\left(\lambda_{\sigma}^{2}+\mu_{\sigma}^{2}+\lambda_{\sigma} \mu_{\sigma}+\right.$ 
$\left.3 \lambda_{\sigma}+3 \mu_{\sigma}\right) / 3+N_{\sigma}^{2} / 3-4 N_{\sigma}$, respectively. These operators, as well as the h.o. $\hat{H}_{0}$ and $\hat{L}^{2}$ terms, are diagonal in the dynamical symmetry basis. Unlike the Elliott quadrupole-quadrupole interaction, $Q_{2}^{E} \cdot Q_{2}^{E}=6 \hat{C}_{S U 3}-3 \hat{L}^{2}$, the $Q_{2} \cdot Q_{2}$ interaction of Eq. (1) breaks $\mathrm{SU}(3)$ symmetry within each h.o. shell since the term $\hat{A}_{0} \hat{B}_{0} \equiv \hat{A}_{0}^{(20)} \hat{B}_{0}^{(02)}=\left(\{\hat{A} \times \hat{B}\}_{0}^{(00)}-\sqrt{5}\{\hat{A} \times \hat{B}\}_{0}^{(22)}\right) / \sqrt{6}$ mixes different $\mathrm{SU}(3)$ irreps.

\section{Partial Dynamical Symmetry in the SSM}

In order to study the action of $Q_{2} \cdot Q_{2}$ within a h.o. shell, we consider the following family of Hamiltonians:

$$
\begin{aligned}
& H\left(\beta_{0}, \beta_{2}\right)=\beta_{0} \hat{A}_{0} \hat{B}_{0}+\beta_{2} \hat{A}_{2} \cdot \hat{B}_{2} \\
& =\frac{\beta_{2}}{18}\left(9 \hat{C}_{S U 3}-9 \hat{C}_{S p 6}+3 \hat{H}_{0}^{2}-36 \hat{H}_{0}\right)+\left(\beta_{0}-\beta_{2}\right) \hat{A}_{0} \hat{B}_{0} .
\end{aligned}
$$

For $\beta_{0}=\beta_{2}$, one recovers the dynamical symmetry, and with the special choice $\beta_{0}=12, \beta_{2}=18$, one obtains $Q_{2} \cdot Q_{2}=H\left(\beta_{0}=12, \beta_{2}=\right.$ $18)+\operatorname{const}(N)-3 \hat{L}^{2}+$ terms coupling different shells, where $\operatorname{const}(N)$ is constant for a given h.o. $N \hbar \omega$ excitation.

It has been shown ${ }^{9}$ that $H\left(\beta_{0}, \beta_{2}\right)$ exhibits partial $\mathrm{SU}(3)$ symmetry of the first kind. Specifically, one finds that among the eigenstates of $H\left(\beta_{0}, \beta_{2}\right)$, there exists a subset of solvable pure-SU(3) states, the $\mathrm{SU}(3) \supset \mathrm{SO}(3)$ classification of which depends on both the Elliott labels $\left(\lambda_{\sigma}, \mu_{\sigma}\right)$ of the starting state and the symplectic excitation $N$. In general, one observes that all L-states in the starting configuration $(N=0)$ are solvable with good $\mathrm{SU}(3)$ symmetry $\left(\lambda_{\sigma}, \mu_{\sigma}\right)$. For excited configurations $(N>0$ and even) one distinguishes between two possible cases:

(a) $\lambda_{\sigma}>\mu_{\sigma}$ : the pure states belong to $(\lambda, \mu)=\left(\lambda_{\sigma}-N, \mu_{\sigma}+N\right)$ and have $L=\mu_{\sigma}+N, \mu_{\sigma}+N+1, \ldots, \lambda_{\sigma}-N+1$ with $N=2,4, \ldots$ subject to $2 N \leq\left(\lambda_{\sigma}-\mu_{\sigma}+1\right)$.

(b) $\lambda_{\sigma} \leq \mu_{\sigma}$ : the special states belong to $(\lambda, \mu)=\left(\lambda_{\sigma}+N, \mu_{\sigma}\right)$ and have $L=\lambda_{\sigma}+N, \lambda_{\sigma}+N+1, \ldots, \lambda_{\sigma}+N+\mu_{\sigma}$ with $N=2,4, \ldots$.

The special states have well-defined symmetry $\mathrm{Sp}(6, \mathrm{R}) \supset \mathrm{SU}(3) \supset \mathrm{SO}(3)$ and are annihilated by $\hat{B}_{0}$. This ensures that they are solvable eigenstates of $H\left(\beta_{0}, \beta_{2}\right)$ with eigenvalues $E(N=0)=0, E(N)=\beta_{2} N\left(N_{\sigma}-\lambda_{\sigma}+\mu_{\sigma}-\right.$ $6+3 N / 2) / 3$ for family (a), and $E(N)=\beta_{2} N\left(N_{\sigma}+2 \lambda_{\sigma}+\mu_{\sigma}-3+3 N / 2\right) / 3$ for family (b). All $0 \hbar \omega$ states are unmixed and span the entire $\left(\lambda_{\sigma}, \mu_{\sigma}\right)$ irrep. In contrast, for the excited levels $(N>0)$, the pure states span only part of the corresponding SU(3) irreps. There are other states at each excited 
level which do not preserve the $\mathrm{SU}(3)$ symmetry and therefore contain a mixture of $\mathrm{SU}(3)$ irreps. All eigenstates respect the $\mathrm{Sp}(6, \mathrm{R})$ and $\mathrm{SO}(3)$ symmetries. The partial $\mathrm{SU}(3)$ symmetry of $H\left(\beta_{0}, \beta_{2}\right)$ is converted into partial dynamical $\mathrm{SU}(3)$ symmetry by adding to it $\mathrm{SO}(3)$ rotation terms which lead to $\mathrm{L}(\mathrm{L}+1)$-type splitting but do not affect the wave functions. The solvable states form rotational bands and since their wave functions are known, one can evaluate the E2 rates between them ${ }^{9}$.

\section{Applications}

To illustrate that the PDS Hamiltonians discussed here are physically relevant, applications to realistic nuclear systems have been considered. Here the results for ${ }^{20} \mathrm{Ne}$ and ${ }^{24} \mathrm{Mg}$ are summarized. In particular, energy spectra and eigenstates of $H_{P D S}=h(N)+\xi H\left(\beta_{0}=12, \beta_{2}=18\right)+\gamma_{2} \hat{L}^{2}+\gamma_{4} \hat{L}^{4}$ are compared to those of $H_{Q \cdot Q}=\hat{H}_{0}-\chi Q_{2} \cdot Q_{2}+d_{2} \hat{L}^{2}+d_{4} \hat{L}^{4}$, where $h(N)$ is a constant for a given $N \hbar \omega$ excitation and contains the h.o. term $\hat{H}_{0}$.

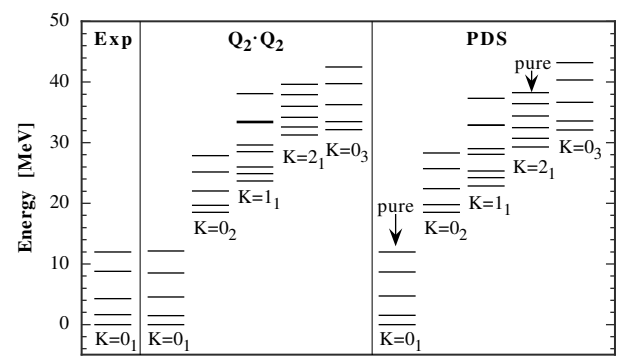

Figure 1. Energy spectra for ${ }^{20} \mathrm{Ne}$. Comparison between experimental values (left), results from a symplectic $8 \hbar \omega$ calculation (center) and a PDS calculation (right). The angular momenta of the positive parity states in the rotational bands are $L=0,2,4, \ldots$ for $\mathrm{K}=0$ and $L=\mathrm{K}, \mathrm{K}+1, \mathrm{~K}+2, \ldots$ otherwise.

\subsection{The ${ }^{20} \mathrm{Ne}$ Example}

In Fig. 1, energy spectra of $H_{P D S}$ are compared to those obtained from an $8 \hbar \omega$ symplectic calculation (labeled $Q_{2} \cdot Q_{2}$ ), and Fig. 2 shows the decomposition for representative $\left(2^{+}\right)$states of the five lowest rotational bands. The PDS Hamiltonian $H_{P D S}$ acts only within one oscillator shell, hence its eigenfunctions do not contain admixtures from different $N \hbar \omega$ configurations. As expected, $H_{P D S}$ has families of pure $\mathrm{SU}(3)$ eigenstates which can be organized into rotational bands, Fig. 1. The ground band belongs entirely to $N=0,(\lambda, \mu)=(8,0)$, and all states of the $\mathrm{K}=2_{1}$ band have quantum labels $N=2,(\lambda, \mu)=(6,2), \kappa=2$, see Fig. 2. A comparison 
with the symplectic case shows that the $N \hbar \omega$ level to which a particular PDS band belongs is also dominant in the corresponding symplectic band. In addition, within this dominant excitation, eigenstates of $H_{P D S}$ and $H_{Q \cdot Q}$ have similar SU(3) distributions; in particular, both Hamiltonians favor the same $(\lambda, \mu) \kappa$ values. Significant differences in the structure of the wave functions appear, however, for the $\mathrm{K}=0_{2}$ resonance band. In the symplectic calculation, this band contains almost equal contributions from the $0 \hbar \omega, 2 \hbar \omega$, and $4 \hbar \omega$ levels, with additional admixtures of $6 \hbar \omega$ and $8 \hbar \omega$ configurations, while in the PDS calculation, it belongs entirely to the $2 \hbar \omega$ level. These structural differences are also evident in the interband transition rates $^{9}$ and reflect the action of the inter-shell coupling terms in Eq. (1). Increasing the strength $\chi$ of $Q_{2} \cdot Q_{2}$ in $H_{Q \cdot Q}$ will also spread the other resonance bands over many $N \hbar \omega$ excitations. The $\mathrm{K}=22_{1}$ band (which is pure in the PDS scheme) is found to resist this spreading more strongly than the other resonances. For physically relevant values of $\chi$, the low-lying bands have the structure shown in Fig. 2.
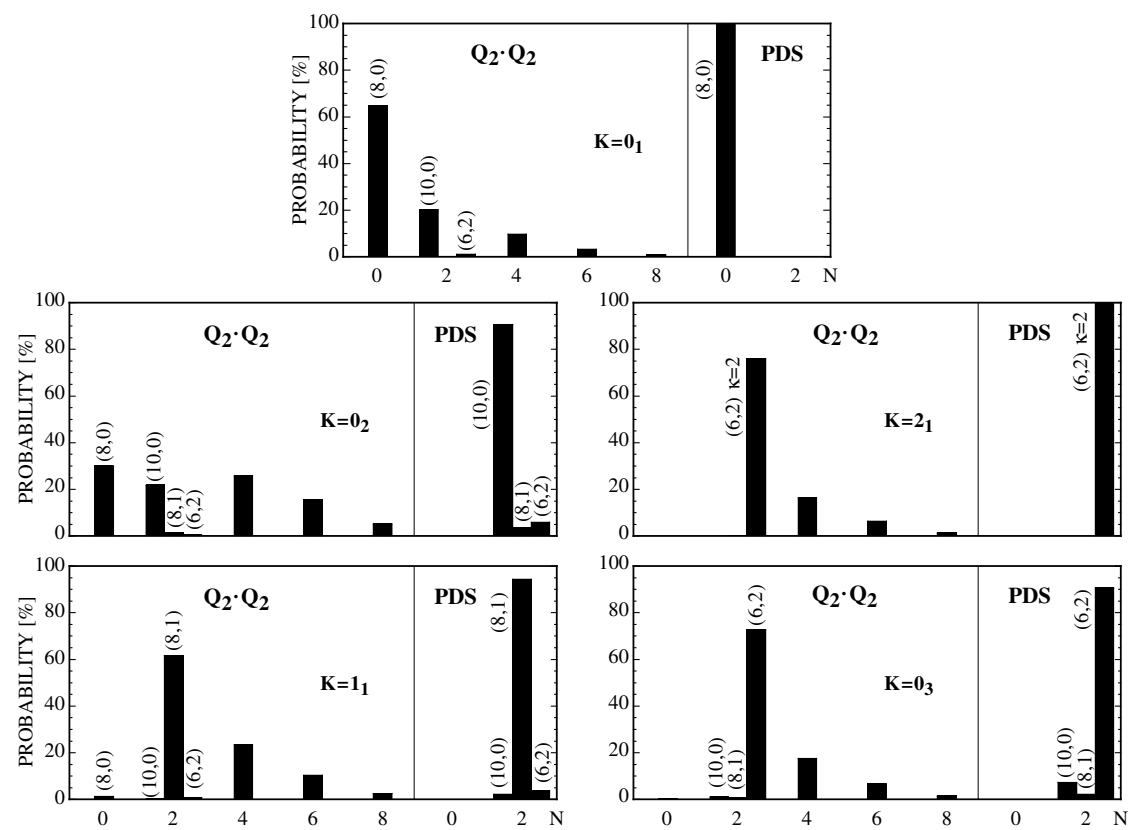

Figure 2. Decomposition for calculated $2^{+}$states of ${ }^{20} \mathrm{Ne}$. Individual contributions from the relevant $\mathrm{SU}(3)$ irreps at the $0 \hbar \omega$ and $2 \hbar \omega$ levels are shown for both a symplectic $8 \hbar \omega$ calculation (denoted $Q_{2} \cdot Q_{2}$ ) and a PDS calculation. In addition, the total strengths contributed by the $N \hbar \omega$ excitations for $N>2$ are given for the symplectic case. 


\subsection{The ${ }^{24} \mathrm{Mg}$ Example}

For the triaxially deformed nucleus ${ }^{24} \mathrm{Mg}$ additional terms $\hat{X}_{3} \equiv\left(\hat{L} \times Q^{E}\right) \cdot \hat{L}$ and $\hat{X}_{4} \equiv\left(\hat{L} \times Q^{E}\right) \cdot\left(\hat{L} \times Q^{E}\right)$ are required in the Hamiltonian in order to reproduce the experimentally observed 'K-band splitting' between the ground and $\gamma$ band of ${ }^{24} \mathrm{Mg}$. Although these extra terms break the partial symmetry, for realistic interaction parameters the amount of symmetry breaking is very small $(\sim 1 \%)$. In Fig. 3, energy spectra of $H_{P D S}^{\prime}=$ $H_{P D S}+c_{3} \hat{X}_{3}+c_{4} \hat{X}_{4}$ and $H_{Q \cdot Q}^{\prime}=H_{Q \cdot Q}+c_{3} \hat{X}_{3}+c_{4} \hat{X}_{4}$ are shown. $H_{P D S}^{\prime}$ has families of pure (and nearly pure) SU(3) eigenstates which can be organized into rotational bands; they are indicated in the figure.
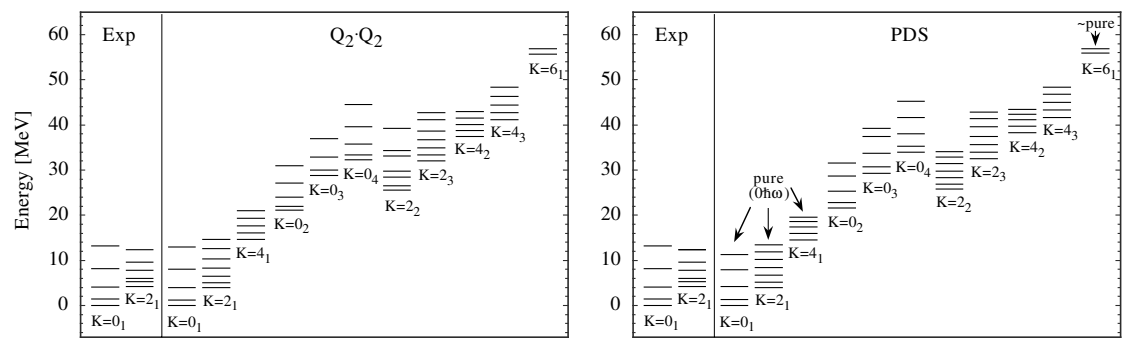

Figure 3. Energy spectra for ${ }^{24} \mathrm{Mg}$. Energies from a PDS calculation (PDS) are compared to symplectic $6 \hbar \omega$ results $\left(Q_{2} \cdot Q_{2}\right)$. Both $0 \hbar \omega$-dominated bands $\left(\mathrm{K}=0_{1}, 2_{1}, 4_{1}\right)$ and some $2 \hbar \omega$ resonance bands are shown. The $K=0_{1}$ and $K=2_{1}$ labels indicate the ground band and $\gamma$ band, respectively.

The results are qualitatively similar to those for ${ }^{20} \mathrm{Ne}$. The PDS Hamiltonian cannot account for intershell correlations, but it is able to reproduce various features of the quadrupole-quadrupole interaction, as can be seen in Fig. 4, where the structure of selected PDS eigenstates is compared to that of the corresponding $Q_{2} \cdot Q_{2}$ eigenstates: PDS eigenfunctions do not contain admixtures from different $N \hbar \omega$ configurations, but belong entirely to one level of excitation. For reasonable interaction parameters, the $N \hbar \omega$ level to which a particular PDS band belongs is also dominant in the corresponding band of exact $Q_{2} \cdot Q_{2}$ eigenstates. Within this dominant excitation, eigenstates of both Hamiltonians have similar SU(3) distributions. Structural differences, nevertheless, do arise and are reflected in the very sensitive interband transition rates ${ }^{9}$. Furthermore, due to the presence of $\hat{X}_{3}$ and $\hat{X}_{4}, H_{P D S}^{\prime}$ is only an approximate PDS Hamiltonian - the $\mathrm{K}=66_{1}$ band has small admixtures from irreps other than $(\lambda, \mu)=(6,6)$. Overall, it may be concluded that PDS eigenstates approximately reproduce the structure of the exact $Q_{2} \cdot Q_{2}$ eigenstates, for both ground and resonance bands. 

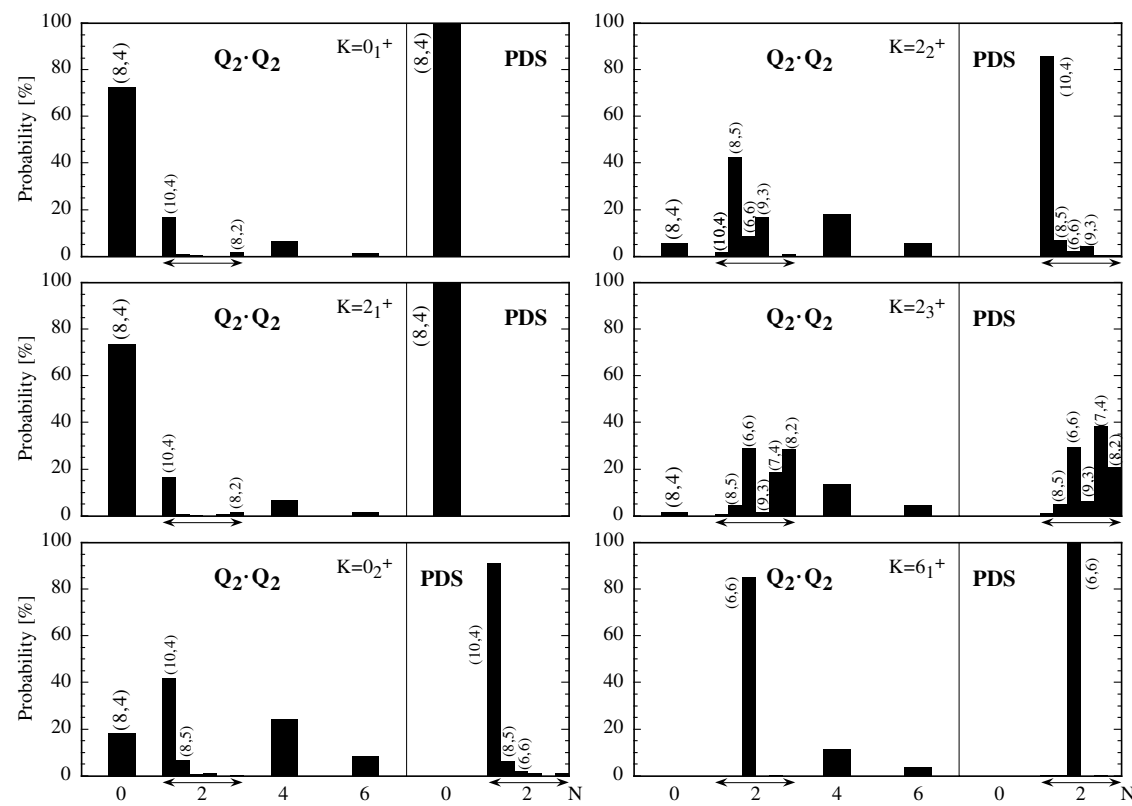

Figure 4. Decompositions for calculated $L^{\pi}=6^{+}$states of ${ }^{24} \mathrm{Mg}$. Eigenstates resulting from a symplectic $6 \hbar \omega\left(Q_{2} \cdot Q_{2}\right)$ calculation are decomposed into their $0 \hbar \omega, 2 \hbar \omega, 4 \hbar \omega, 6 \hbar \omega$ components. At the $0 \hbar \omega$ and $2 \hbar \omega$ levels, contributions from individual $\mathrm{SU}(3)$ irreps are shown, for higher excitations $(N>2)$ only the summed strengths are given. Eigenstates of $H_{P D S}^{\prime}$ belong entirely to one $N \hbar \omega$ level of excitation, here $0 \hbar \omega$ or $2 \hbar \omega$; members of the $\mathrm{K}=0_{1}$ and $\mathrm{K}=2_{1}$ bands are pure and $\mathrm{K}=6_{1}$ states are very nearly (>99\%) pure.

\section{Concluding Remarks}

The notion of partial dynamical symmetry extends and complements the familiar concepts of exact and dynamical symmetry. It allows one to remove undesired constraints from an algebraic theory while preserving some of the useful aspects of a dynamical symmetry. As a result, the effects of symmetry breaking can be studied in a controlled manner and new insights into dynamics of the system under consideration are gained.

The work presented here focuses on a family of PDS Hamiltonians which are closely related to the deformation-inducing quadrupole-quadrupole interaction. For a particular parametrization, the PDS Hamiltonians take a form that is intermediate between the full quadrupole-quadrupole interaction, which couples states belonging to different harmonic oscillator shells, and the Elliott quadrupole-quadrupole interaction, which acts only within a shell. The intermediate scheme considered here extends the Elliott picture in that it includes (specific) $\mathrm{SU}(3)$ symmetry-breaking contributions. 
At the same time, it is simpler than the full collective picture since it does not allow for mixing between different oscillator shells.

The PDS scheme sheds light on the in-shell behavior of the quadrupolequadrupole interaction. For example, the symplectic model predicts the existence of states that are primarily dominated by one $N \hbar \omega$ level of excitation as well as states that contain strong multi-shell correlations ${ }^{\mathrm{a}}$. The states that resist the deformation-induced spreading over several $N \hbar \omega$ levels of excitation the strongest are those for which the associated PDS structure exhibits good (or almost good) SU(3) symmetry.

\section{Acknowledgments}

It has been a pleasure to present this work on the occasion of Jerry Draayer's 60 th birthday. The research follows a tradition of using symmetry principles to gain insights into physical systems. Throughout his career, Jerry Draayer has contributed much to this tradition and has inspired many to seek the simplicity, symmetry, and beauty hidden within complex physical systems. The work presented here was carried out in collaboration with A. Leviatan (Hebrew University, Jerusalem). It was performed in part under the auspices of the U. S. Department of Energy by the University of California, Lawrence Livermore National Laboratory, under contract No. W-7405-Eng-48.

\section{References}

1. C. Bahri, D.J. Rowe, and W. Wijesundera, Phys. Rev. C 58, 1539 (1998); C. Bahri and D.J. Rowe, Nucl. Phys. A 662, 125 (2000); D.J. Rowe, Embedded representations and quasi-dynamical symmetry, contribution in this volume.

2. See J.N. Ginocchio's and P. Van Isacker's contributions in this volume and references therein.

3. Y. Alhassid and A. Leviatan, J. Phys. A25, L1265 (1992); A. Leviatan, Phys. Rev. Lett. 77, 818 (1996).

4. P. Van Isacker, Phys. Rev. Lett. 83, 4269 (1999).

5. A. Leviatan and P. Van Isacker, Phys. Rev. Lett. 89, 222501 (2002).

6. G. Rosensteel and D.J. Rowe, Phys. Rev. Lett. 38, 10 (1977); Ann. Phys. 126, 343 (1980); D.J. Rowe, Rep. Prog. Phys. 48, 1419 (1985).

7. J.P. Elliott, Proc. Roy. Soc. A245, 128 (1958); A245, 562 (1958).

8. J. Escher and J.P. Draayer, J. Math. Phys. 39, 5123 (1998).

9. J. Escher and A. Leviatan, Phys. Rev. Lett. 84, 1866 (2000); Phys. Rev. C65, 24305 (2002).

10. C. Bahri et al., Phys. Lett. B234, 430 (1990).

${ }^{a}$ This result extends the findings of Bahri et al. ${ }^{10}$, who studied the structure of the giant quadrupole resonance in ${ }^{24} \mathrm{Mg}$. 